\title{
STATISTICAL ANALYSIS OF FAST FLUCTUATING RANDOM SIGNALS WITH ARBITRARY-FUNCTION ENVELOPE AND UNKNOWN PARAMETERS
}

We find a new expression for the solving statistics of fast fluctuating Gaussian pulses with arbitrary-function envelope, based on which we can receive much simpler processing algorithms of random signals with unknown parameters in comparison with available analogues. For example, the synthesis and analysis of maximum likelihood detector and measurer of a high-frequency random pulse with unknown time parameter is carried out. The asymptotically exact expressions for detection and estimation characteristics including anomalous effects are presented. By methods of statistical computer modeling the adequacy of the considered analytical approach of the statistical analysis of random pulsed signals is corroborated, the working capacity of offered detector and measurer is established, and applicability borders of asymptotically exact formulas for their characteristics are also defined.

Keywords: Fast fluctuating random signal, maximum likelihood method, solving statistics, signal detection and estimation, local Markov approximation method, statistical modeling.

\section{Introduction}

In a number of practical appendices of statistical radio engineering and radio physics it is necessary to solve a processing problem of random pulsed signals with unknown parameters against hindrances. In [ 1 - 5, etc.] the detection and measurement tasks of random Gaussian pulses with rectangular or close to the rectangular envelope are considered in the conditions of various parametrical prior uncertainty. However, a received signal waveform can essentially differ from the rectangular often enough. Thereupon, it is of interest to find the optimal receiver structure for random Gaussian pulse with arbitrary-function envelope.

\section{The output signal of the maximum likelihood} receiver of a random Gaussian pulse

As a random Gaussian pulse with arbitrary-function envelope, we will understand a multiplicative combination taking a form of:

$\mathrm{s}(\mathrm{t})=\xi(\mathrm{t}) \mathrm{f}\left[\gamma\left(\mathrm{t}-\lambda_{0}\right)\right] \mathrm{I}\left(\frac{\mathrm{t}-\lambda_{0}}{\tau_{0}}\right)$,

$I(x)=\left\{\begin{array}{r}1,|x| \leq 1 / 2 \\ 0,|x|>1 / 2\end{array}\right.$
Here $\lambda_{0}$ is the appearance time, $\tau_{0}$ is the duration of a pulse, $\xi(\mathrm{t})$ is the stationary Gaussian random process, $f(t)$ is the determined function (envelope), which is describing the pulse form and is normalized so that $\max f(t)=1$, and $\gamma$ is a scaling multiplier. We will designate $\mathrm{a}_{0}=\langle\xi(\mathrm{t})\rangle$ as mathematical expectation,

$\mathrm{B}_{\xi}\left(\mathrm{t}_{2}-\mathrm{t}_{1}\right)=\left\langle\left[\xi\left(\mathrm{t}_{1}\right)-\left\langle\xi\left(\mathrm{t}_{1}\right)\right\rangle\right]\left[\xi\left(\mathrm{t}_{2}\right)-\left\langle\xi\left(\mathrm{t}_{2}\right)\right\rangle\right]\right\rangle$ - as covariance function and

$\mathrm{G}_{\xi}(\omega)=\int_{-\infty}^{\infty} \mathrm{B}_{\xi}(\mathrm{t}) \exp (-\mathrm{j} \omega \tau) \mathrm{d} \tau$ - as spectral density (SD) of the process $\xi(\mathrm{t})$.

Let us consider that fluctuations $\xi(\mathrm{t})$ are "fast", that is, the pulse duration $\tau_{0}$ and the characteristic changing time $\Delta t$ of the function $f(t)$ essentially exceed the correlation time of the process $\xi(\mathrm{t})$, so the following conditions are satisfied:

$\tau_{0}>>2 \pi / \Omega_{0}, \Delta \mathrm{t}>>2 \pi / \Omega_{0}$.

Here $\Omega_{0}$ - bandwidth of the process $\xi(\mathrm{t})$. Hindrances and $n(t)$ registration errors will be approximated by Gaussian white noise with the one-sided SD $\mathrm{N}_{0}$.

\footnotetext{
* ${ }^{1}$ Oleg V. Chernoyarov, ${ }^{2}$ Martin Vaculik, ${ }^{3}$ Armen Shirikyan, ${ }^{4}$ Alexandra V. Salnikova ${ }^{1}$ National Research University "Moscow Power Engineering Institute", Moscow, Russia ${ }^{2}$ Department of Telecommunications and Multimedia, University of Zilina, Slovakia ${ }^{3}$ University of Cergy-Pontoise, Cergy-Pontoise, France

${ }^{4}$ Voronezh State University of Architecture and Civil Engineering, Voronezh, Russia E-mail: o_v_ch@mail.ru
} 
For the signal (1) detection and measurement, we will use a maximum likelihood method [1,6 and 7]. According to [6], the maximum likelihood receiver (MLR) should form the logarithm of the functional of likelihood ratio (FLR) $L(\vec{\vartheta})$ as function of current values $\vec{\vartheta}$ of set of unknown parameters $\vec{\vartheta}_{0}$ (let us accept for further that true value of parameter is designated with the subindex "0", and its current value - without the subindex). Any signal (1) parameters (appearance time, duration, mathematical expectation, etc) can be vector $\vec{\vartheta}_{0}$ components.

Let the realization $\mathrm{x}(\mathrm{t})=\mathrm{s}(\mathrm{t})+\mathrm{n}(\mathrm{t})$ or $\mathrm{x}(\mathrm{t})=\mathrm{n}(\mathrm{t})$ be received to the MLR input. Then, in concordance with [1 and 4], the logarithm of FLR can be presented as

$\mathrm{L}(\vec{\vartheta})=\frac{1}{\mathrm{~N}_{0}} \int_{0}^{\mathrm{T}} \int_{0}^{\mathrm{T}} \mathrm{x}\left(\mathrm{t}_{1}\right) \mathrm{x}\left(\mathrm{t}_{2}\right) \mathrm{Q}\left(\mathrm{t}_{1}, \mathrm{t}_{2}\right) \mathrm{dt}_{1} \mathrm{dt}_{2}+\int_{0}^{\mathrm{T}} \mathrm{x}(\mathrm{t})$
$\mathrm{V}(\mathrm{t}) \mathrm{dt}-\frac{1}{2} \int_{0}^{\mathrm{T}} \mathrm{a}_{\mathrm{s}}(\mathrm{t}) \mathrm{V}(\mathrm{t}) \mathrm{dt}-\frac{1}{2} \int_{0}^{1} \mathrm{~d} \chi \int_{0}^{\mathrm{T}} \tilde{\mathrm{Q}}(\mathrm{t}, \mathrm{t}, \boldsymbol{\chi}) \mathrm{dt}$.

Here, $\mathrm{a}_{s}(\mathrm{t})=\langle\mathrm{s}(\mathrm{t})\rangle=\mathrm{af}[\gamma(\mathrm{t}-\lambda)] \mathrm{I}[(\mathrm{t}-\lambda) / \mathrm{t}]$,

$\mathrm{V}(\mathrm{t})=\frac{2}{\mathrm{~N}_{0}}\left[\mathrm{a}_{\mathrm{s}}(\mathrm{t})-\int_{0}^{\mathrm{T}} \mathrm{a}_{\mathrm{s}}\left(\mathrm{t}^{\prime}\right) \mathrm{Q}\left(\mathrm{t}, \mathrm{t}^{\prime}\right) \mathrm{dt^{ \prime }}\right]$

$\mathrm{Q}\left(\mathrm{t}_{1}, \mathrm{t}_{2}\right)=\tilde{\mathrm{Q}}\left(\mathrm{t}_{1}, \mathrm{t}_{2}, 1\right)$, and function $\tilde{\mathrm{Q}}\left(\mathrm{t}_{1}, \mathrm{t}_{2}, \boldsymbol{\chi}\right)$ satisfies the following integral equation:

$\frac{\mathrm{N}_{0}}{2} \tilde{\mathrm{Q}}\left(\mathrm{t}_{1}, \mathrm{t}_{2}, \chi\right)+\chi \int_{0}^{\mathrm{T}} \tilde{\mathrm{Q}}\left(\mathrm{t}_{1}, \mathrm{t}_{2}, \chi\right) \mathrm{B}_{\mathrm{s}}\left(\mathrm{t}, \mathrm{t}_{2}\right) \mathrm{dt}=\mathrm{B}_{\mathrm{s}}\left(\mathrm{t}_{1}, \mathrm{t}_{2}\right)$,

where

$\mathrm{B}_{\mathrm{s}}\left(\mathrm{t}_{1}, \mathrm{t}_{2}\right)=\left\langle\left[\mathrm{s}\left(\mathrm{t}_{1}\right)-\mathrm{a}_{\mathrm{s}}\left(\mathrm{t}_{1}\right)\right]\left[\mathrm{s}\left(\mathrm{t}_{2}\right)-\mathrm{a}_{\mathrm{s}}\left(\mathrm{t}_{2}\right)\right]\right\rangle=$

$=B_{\xi}\left(t_{2}-t_{1}\right) f\left[\gamma\left(t_{1}-\lambda\right)\right] f\left[\gamma\left(t_{2}-\lambda\right)\right] I\left(\frac{t_{1}-\lambda}{\tau}\right) I\left(\frac{t_{2}-\lambda}{\tau}\right)^{(6)}$

is the covariance function of signal $s(t)$. If some signal (1) parameter is known, then in Eqs. (3)-(6) its true value should be used instead of current value.

We will try solution of Eq. (5) in a form structurally similar to Eq. (6), i.e.

$\tilde{Q}\left(t_{1}, t_{2}, \chi\right)=\tilde{Q}_{0}\left(t_{1}, t_{2}, \chi\right) f\left[\gamma\left(t_{1}-\lambda\right)\right] f\left[\gamma\left(t_{2}-\lambda\right)\right]$

$\mathrm{I}\left[\left(\mathrm{t}_{1}-\lambda\right) / \tau\right] \mathrm{I}\left[\left(\mathrm{t}_{2}-\lambda\right) / \tau\right]$

Substituting Eqs. (6), (7) in Eq. (5), we come to the equation of a form

$$
\begin{aligned}
& \frac{\mathrm{N}_{0}}{2} \tilde{Q}_{0}\left(\mathrm{t}_{1}, \mathrm{t}_{2}, \chi\right)+\chi \int_{\lambda-\tau / 2}^{\lambda+\tau / 2}[\gamma(\mathrm{t}-\lambda)] \tilde{\mathrm{Q}}_{0}\left(\mathrm{t}_{1}, \mathrm{t}, \chi\right) . \\
& \mathrm{B}_{\xi}\left(\mathrm{t}_{2}-\mathrm{t}\right) \mathrm{dt}=\mathrm{B}_{\xi}\left(\mathrm{t}_{2}-\mathrm{t}_{1}\right)
\end{aligned}
$$

Solving Eq. (8) by Fourier transformation method [8], with a variable $\Delta=\mathrm{t}_{2}-\mathrm{t}_{1}$ and taking into account the ratios (2), we come to
$\tilde{Q}_{0}\left(t_{1}, t_{2}, \chi\right)=\frac{1}{2 \pi} \int_{-\infty}^{\infty} \frac{G_{\xi}(\omega) \exp \left[j \omega\left(t_{2}-t_{1}\right)\right]}{N_{0} / 2+\chi f^{2}\left[\gamma\left(t_{1}-\lambda\right)\right] G_{\xi}(\omega)} d \omega$

In terms of Eq. (9), we specify expressions for the summands entering the logarithm of FLR (3). Using Eqs. (7), (9), we can present last summand in Eq. (3) as

$$
\begin{aligned}
& \frac{1}{2} \int_{0}^{1} \mathrm{~d} \chi \int_{0}^{\mathrm{T}} \tilde{\mathrm{Q}}(\mathrm{t}, \mathrm{t}, \boldsymbol{\chi}) \mathrm{dt}=\frac{1}{4 \pi} \int_{-\tau / 2}^{\tau / 2} \\
& \int^{\infty} \ln \left[1+\frac{2 \mathrm{f}^{2}(\gamma \mathrm{t}) \mathrm{G}_{\xi}(\omega)}{\mathrm{N}_{0}}\right] \mathrm{d} \omega \mathrm{dt}
\end{aligned}
$$

Further, we set $\chi=1$ in Eq. (9) and then substitute Eq. (9) into Eq. (7), and Eq. (7) into Eq. (4), and so, after corresponding transformations for the function $V(t)$, we come to

$$
\mathrm{V}(\mathrm{t}) \approx \frac{2 \mathrm{a}}{\mathrm{N}_{0}\left[1+\mathrm{qf}^{2}(\gamma(\mathrm{t}-\lambda))\right]} \mathrm{f}[\gamma(\mathrm{t}-\lambda)] \mathrm{I}\left(\frac{\mathrm{t}-\lambda}{\tau}\right),
$$

where $q=2 G_{\xi}(0) / N_{0}$. Now, after substituting Eq. (9) into Eq. (7) and then Eqs. (7), (10), (11) into Eq. (3), we receive the following expression for the logarithm of FLR for the random Gaussian pulse with arbitrary-function envelope:

$\mathrm{L}(\vec{\vartheta})=\mathrm{L}_{1}(\vec{\vartheta})+\mathrm{L}_{2}(\vec{\vartheta})-\mathrm{L}_{3}(\vec{\vartheta})$

$\mathrm{L}_{1}(\vec{\vartheta})=\frac{1}{\mathrm{~N}_{0}} \int_{\lambda-\tau / 2}^{\lambda+\tau / 2} \mathrm{x}\left(\mathrm{t}_{1}\right) \mathrm{x}\left(\mathrm{t}_{2}\right) \mathrm{f}\left[\gamma\left(\mathrm{t}_{1}-\lambda\right)\right]$

$\mathrm{f}\left[\gamma\left(\mathrm{t}_{2}-\lambda\right)\right] \mathrm{Q}_{0}\left(\mathrm{t}_{1}, \mathrm{t}_{2}\right) \mathrm{dt}_{1} \mathrm{dt}_{2}$

$\mathrm{L}_{2}(\vec{\vartheta})=\frac{2 \mathrm{a}}{\mathrm{N}_{0}}\left\{\int_{\lambda-\tau / 2}^{\lambda+\tau / 2} \frac{\mathrm{f}[\gamma(\mathrm{t}-\lambda)]_{\mathrm{X}}(\mathrm{t}) \mathrm{dt}}{1+\mathrm{qf}^{2}[\gamma(\mathrm{t}-\lambda)]}-\frac{\mathrm{a}}{2}\right.$

$\left.\int_{-\tau / 2}^{\tau / 2} \frac{\mathrm{f}^{2}(\gamma \mathrm{t}) \mathrm{dt}}{1+\mathrm{qf}^{2}(\gamma \mathrm{t})}\right\}$

$\mathrm{L}_{3}(\vec{\vartheta})=\frac{1}{4 \pi} \int_{-\tau / 2}^{\tau / 2} \int_{-\infty}^{\infty} \ln \left[1+\frac{\mathrm{f}^{2}(\gamma \mathrm{t}) \mathrm{G}_{\xi}(\omega)}{\mathrm{N}_{0}}\right] \mathrm{d} \omega \mathrm{dt}$.

Here, $\mathrm{Q}_{0}\left(\mathrm{t}_{1}, \mathrm{t}_{2}\right)=\tilde{\mathrm{Q}}_{0}\left(\mathrm{t}_{1}, \mathrm{t}_{2}, 1\right)$.

The formula (12) is essentially simplified if $\mathrm{SD}_{\xi}(\omega)$ allows for rectangular approximation [4]:

$\mathrm{G}_{\xi}(\omega)=\left(\mathrm{d}_{0} / 2\right) \mathrm{I}\left(\omega / \Omega_{0}\right)$

for low-frequency (LF) process $\xi(t)$ and

$\mathrm{G}_{\xi}(\omega)=\left(\mathrm{d}_{0} / 2\right)\left\{\mathrm{I}\left[\left(\mathrm{v}_{0}-\omega\right) / \Omega_{0}\right]+\mathrm{I}\left[\left(\mathrm{v}_{0}+\omega\right) / \Omega_{0}\right]\right\}$

for high-frequency (HF) process $\xi(\mathrm{t})$ with a central frequency $\nu_{0}$. Here $\mathrm{d}_{0}$ is SD magnitude. Such kind of approximation of the SD form can be used if real SD rapidly decreases outside of the bandwidth $\Omega_{0}$. With the realization of Eq. (13) or (14), the first summand in Eq. (12) can be written down in a form: 
$\mathrm{L}_{1}(\vec{\vartheta})=\frac{\mathrm{d}}{\mathrm{N}_{0}} \int_{-\infty}^{\infty} \tilde{y}^{2}(\mathrm{t}) \mathrm{dt}$

where

$\tilde{y}(t)=\int^{\infty} \tilde{x}\left(t^{\prime}\right) h\left(t-t^{\prime}\right) d t^{\prime}$,

$\tilde{\mathrm{x}}(\mathrm{t})=\frac{\mathrm{x}(\mathrm{t})}{\sqrt{\mathrm{N}_{0}+\mathrm{df}^{2}[\gamma[-\lambda]}} \mathrm{f}[\gamma(\mathrm{t}-\lambda)] \mathrm{I}\left(\frac{\mathrm{t}-\lambda}{\tau}\right)$,

$h(t)$ is the function, the spectrum $\mathrm{H}(\omega)$ of which satisfies the condition

$|\mathrm{H}(\omega)|^{2}=2 \mathrm{G}_{\xi}(\omega) / \mathrm{d}_{0}$,

and $d$ is current value of the SD magnitude (in a case when the parameter $\mathrm{d}_{0}$ is unknown).

As follows from the last parity, if inequalities (2) hold, then the duration of the pulse response $h(t)$ is much less than the signal $\mathrm{s}(\mathrm{t})(1)$ duration and the characteristic changing time $\Delta t$ of the function $f(t)$. Then the filter response $\tilde{y}(t)$ (16) allows representation:

$$
\begin{aligned}
& \tilde{y}(t) \approx \frac{y(t)}{\sqrt{N_{0}+d f^{2}[\gamma(t-\lambda)]}} f[\gamma(t-\lambda)] I\left(\frac{t-\lambda}{\tau}\right), \\
& y(t)=\int_{-\infty}^{\infty} x\left(t^{\prime}\right) h\left(t-t^{\prime}\right) d t^{\prime} .
\end{aligned}
$$

Indeed, the duration of the transient processes in the filter with the pulse response $h(t)$ (16) has an order of magnitude $2 \pi / \Omega$. Therefore, the right and the left parts of the approximate equality (18) can differ essentially only in time intervals of an order $2 \pi / \Omega$. in the neighborhood of the points $t=\lambda-\tau / 2$ and $\mathrm{t}=\lambda+\tau / 2$. Still we can neglect this difference considering that Eq. (18) enters the logarithm of FLR under integral sign while executing ratios (2). Substituting Eq. (18) into Eq. (15) we have:

$\mathrm{L}_{1}(\vec{\vartheta})=\frac{\mathrm{d}}{\mathrm{N}_{0}} \int_{\lambda-\tau / 2}^{\lambda+\tau / 2} \frac{\mathrm{f}^{2}[\gamma(\mathrm{t}-\lambda)] \mathrm{y}^{2}(\mathrm{t})}{\mathrm{N}_{0}+\mathrm{df}^{2}[\gamma(\mathrm{t}-\lambda)]} \mathrm{dt}$.

In view of Eqs. (13), (14), we will transform summand $\mathrm{L}_{3}(\vec{\vartheta})$ into a form:

$\mathrm{L}_{3}(\vec{\vartheta})=\frac{\varepsilon \Omega}{4 \pi} \int_{-\tau / 2}^{\tau / 2} \ln \left[1+\frac{\mathrm{d}}{\mathrm{N}_{0}} \mathrm{f}^{2}(\gamma \mathrm{t})\right] \mathrm{dt}$,

where $\varepsilon=1$ or $\varepsilon=2$ for LF or HF process $\xi(\mathrm{t})$ correspondingly.

Applying Eqs. (19), (20) in Eq. (12) for the logarithm of FLR for the Gaussian pulse with the arbitrary-function envelope and band SD, we receive:

$$
\begin{aligned}
& \mathrm{L}(\vec{\vartheta})=\frac{\mathrm{q}}{\mathrm{N}_{0}} \int_{\lambda-\tau / 2}^{\lambda+\tau / 2} \frac{\mathrm{f}^{2}[\gamma(\mathrm{t}-\lambda)] \mathrm{y}^{2}(\mathrm{t})}{1+\mathrm{qf}^{2}[\gamma(\mathrm{t}-\lambda)]} \mathrm{dt}+\frac{2 \mathrm{a}}{\mathrm{N}_{0}} \\
& \int_{\lambda-\tau / 2}^{\lambda+\tau / 2} \frac{\mathrm{f}[\gamma(\mathrm{t}-\lambda)]_{\mathrm{X}}(\mathrm{t})}{1+\mathrm{qf}^{2}[\gamma(\mathrm{t}-\lambda)]} \mathrm{dt}-\mathrm{L}_{0}(\vec{\vartheta}),
\end{aligned}
$$

$\mathrm{L}_{0}(\vec{\vartheta})=\frac{\mathrm{a}^{2}}{\mathrm{~N}_{0}} \int_{-\tau / 2}^{\tau / 2} \frac{\mathrm{f}^{2}(\gamma \mathrm{t})}{1+\mathrm{qf}^{2}(\gamma \mathrm{t})} \mathrm{dt}+\frac{\Omega}{4 \pi}$

$\int_{-\tau / 2}^{\tau / 2} \ln \left[1+\mathrm{qf}^{2}(\gamma \mathrm{t})\right] \mathrm{dt}$

if process $\xi(\mathrm{t})$ is $\mathrm{LF}$, and

$L(\vec{\vartheta})=\frac{q}{N_{0}} \int_{\lambda-\tau / 2}^{\lambda+\tau / 2} \frac{f^{2}[\gamma(t-\lambda)] y^{2}(t)}{1+\mathrm{qf}^{2}[\gamma(\mathrm{t}-\lambda)]} \mathrm{dt}-\mathrm{L}_{0}(\vec{\vartheta})$,

$\mathrm{L}_{0}(\vec{\vartheta})=\frac{\Omega}{2 \pi} \int_{-\tau / 2}^{\tau / 2} \ln \left[1+\mathrm{qf}^{2}(\gamma \mathrm{t})\right] \mathrm{dt}$,

if process $\xi(t)$ is HF. Here, $q=d / N_{0}$.

\section{Properties of the output signal of the maximum likelihood receiver of a random Gaussian pulse with unknown appearance time}

For the definiteness of the further calculations we will refer to the process $\xi(\mathrm{t})$ as to a bandpass HF random process (14) and we will select pulse appearance time $\lambda_{0}$ as unknown parameter (other signal parameters are supposed to be known).

In the presence of a useful signal (1), (14), the term

$\mathrm{M}(\lambda)=\int_{\lambda-\tau_{0} / 2}^{\lambda+\tau_{0} / 2} \frac{\mathrm{f}^{2}[\gamma(\mathrm{t}-\lambda)] \mathrm{y}^{2}(\mathrm{t})}{1+\mathrm{q}_{0} \mathrm{f}^{2}[\gamma(\mathrm{t}-\lambda)]} \mathrm{dt}$

of the functional $\mathrm{L}(\vec{\vartheta})=\mathrm{L}(\boldsymbol{\lambda})$, depending on the realization of the observable data $x(t)$, can be presented as the sum of regular and noise functions [6]: $\mathrm{M}(\lambda)=\mathrm{S}(1)+\mathrm{N}(1)$, where $\mathrm{S}(1)=\langle\mathrm{M}(\lambda)\rangle$ is regular function, $\mathrm{N}(1)=\mathrm{M}(\lambda)-\langle\mathrm{M}(\lambda)\rangle$ is noise function, $\mathrm{q}_{0}=\mathrm{d}_{0} / \mathrm{N}_{0}, \mathrm{l}=\lambda / \tau_{0}$ is current value of the dimensionless parameter $1_{0}=\lambda_{0} / \tau_{0}$, and the averaging is executed through all possible realizations $\mathrm{x}(\mathrm{t})$ with the fixed $\lambda_{0}$ value. While executing the ratios (2), for regular function and covariance function of noise function we receive

$S(1)=\mu d_{0} C\left(1-1_{0}\right)+S n$,

$\left\langle\mathrm{N}\left(1_{1}\right) \mathrm{N}\left(1_{2}\right)\right\rangle=\mu \mathrm{N}_{0}^{2}\left[\mathrm{R}_{1}\left(1_{1}, 1_{2}, 1_{0}\right)+\mathrm{R}_{2}\left(1_{1}, l_{2}\right)\right]$,

where

$C(x)=\int_{1 / 2+\max (0, x)}^{1 / 2+\min (0, x)} \mathrm{g}_{21}(\tilde{\mathrm{t}}-\mathrm{x}) \mathrm{f}^{2}(\tilde{\gamma} \tilde{\mathrm{t}}) \mathrm{d} \tilde{\mathrm{t}}$,

$\mathrm{S}_{N}=\mu \mathrm{N}_{0} \mathrm{G}_{21}(1 / 2)$,

$\mathrm{R}_{1}\left(1_{1}, 1_{2}, 1_{0}\right)=\int_{1 / 2+\max \left(0, l_{1}-l_{0}, l_{2}-l_{0}\right)}^{1 / 2+\min \left(0, l_{1}-l_{0}, l_{2}-l_{0}\right)} \mathrm{g}_{21}\left(\tilde{\mathrm{t}}-1_{1}+l_{0}\right)$,

$\mathrm{g}_{21}\left(\tilde{\mathrm{t}}-\mathrm{l}_{2}+\mathrm{l}_{0}\right)\left[\left(1+\mathrm{q}_{0} \mathrm{f}^{2}(\tilde{\gamma} \tilde{\mathrm{t}})\right)^{2}-1\right] \mathrm{d} \tilde{\mathrm{t}}$

$\mathrm{R}_{1}\left(1_{1}, 1_{2}\right)=\int_{1 / 2+\max \left(l_{1}, l_{2}\right)}^{1 / 2+\min \left(l_{1}, l_{2}\right)} \mathrm{g}_{21}\left(\tilde{\mathrm{t}}-1_{1}\right) \mathrm{g}_{21}\left(\tilde{\mathrm{t}}-1_{2}\right) \mathrm{d} \tilde{\mathrm{t}}$, 
$\mathrm{x} \in\left[\mathrm{X}_{1}, \mathrm{X}_{2}\right]$, possesses drift coefficient

$\mathrm{g}_{n m n}(\tilde{\mathrm{t}})=\frac{\mathrm{f}^{m}(\tilde{\gamma} \tilde{\mathrm{t}})}{\left[1+\mathrm{q}_{0} \mathrm{f}^{2}(\tilde{\gamma} \mathrm{t})\right]^{n}}$,

$G_{m n}(\tilde{t})=\int_{-\tilde{t}}^{\tilde{t}} g_{m n}(\tilde{t}) d \tilde{t}$,

$\mu=\tau_{0} \Omega_{0} / 2 \pi, \tilde{\gamma}=\gamma \tau_{0}, \tilde{\mathrm{t}}=\mathrm{t} / \tau_{0}$.

If the useful signal (1), (14) is absent, then, both for regular function and covariance function of noise function of the functional (22), we have

$\mathrm{S}(1)=\mathrm{S}_{N},\left\langle\mathrm{~N}\left(\mathrm{l}_{1}\right) \mathrm{N}\left(\mathrm{l}_{2}\right)\right\rangle=\mu \mathrm{N}_{0}^{2} \mathrm{R}_{2}\left(l_{1}, \mathrm{l}_{2}\right)$.

\section{The appearance time estimate of the random Gaussian pulse}

Let us state that the signal (1), (14) is present with the probability 1 . Then, according to [1 and 6], the maximum likelihood estimate (MLE) $\lambda_{m}$ of appearance time $\lambda_{0}$ will be defined as

$$
\lambda_{m}=\underset{\lambda \in\left[\Lambda_{1}, \Lambda_{2}\right]}{\arg \sup } \mathrm{L}(\boldsymbol{\lambda})=\underset{\lambda \in\left[\Lambda_{1}, \Lambda_{2}\right]}{\arg \sup } \mathrm{M}(\boldsymbol{\lambda})
$$

The block diagram of the measurer (26) is shown in Fig. 1 where the designations are the following: 1 is the switch that is open for time $\left[\Lambda_{1}-\tau_{0} / 2, \Lambda_{2}+\tau_{0} / 2\right] ; 2$ is a filter with transfer function $\mathrm{H}(\omega)$ (14), (17); 3 is the squarer; 4 is the filter matched with the signal $\mathrm{f}^{2}(\gamma \mathrm{t}) \mathrm{I}\left(\mathrm{t} / \tau_{0}\right) /\left[1+\mathrm{q}_{0} \mathrm{f}^{2}(\gamma \mathrm{t})\right] ; 5$ is the extremator that fixes the location of the greatest maximum of the input signal as an estimate $\lambda_{m}$. As appears from Fig. 1, synthesized optimal receiver of $\mathrm{HF}$ random pulse with arbitrary-function envelope and unknown appearance time has a single-channel structure, unlike alternative multichannel variants presented in the current researches [2, etc.].

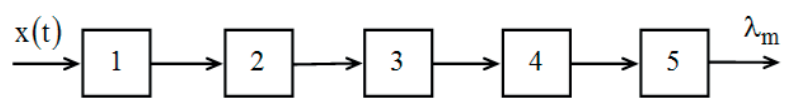

Fig. 1 The optimal measurer of appearance time of HF random pulse

Let us find the characteristics of MLE $\lambda_{m}$. During the analysis process, we find it expedient to divide all the estimates into the two classes: reliable and anomalous [6]. The estimate $1_{m}=\lambda_{m} / \tau_{0}$ is reliable, if it is within the interval limits $\Gamma \equiv\left[1_{0}-1,1_{0}+1\right]$ where the regular function (23) is distinct from $\mathrm{S}_{N}$. If MLE $l_{m}$ is out of an interval $\Gamma_{S}$, i.e. $1_{m} \in \Gamma_{N}=\Gamma \backslash \Gamma_{S}, \Gamma \equiv\left[\tilde{\Lambda}_{1}, \tilde{\Lambda}_{2}\right]$, $\tilde{\Lambda}_{1,2}=\Lambda_{1,2} / \tau_{0}$, then the estimate and the corresponding estimate error are designated as anomalous [6]. It is necessary to consider the anomalous errors if the length of the prior interval $\left[\Lambda_{1}, \Lambda_{2}\right]$ of the possible values of appearance time $\lambda_{0}$ is much greater than signal (1) duration $\tau_{0}$, i.e. the following condition holds

$\mathrm{m}=\tilde{\Lambda}_{2}-\tilde{\Lambda}_{1}>>1$.

According to [6], while executing ratio (27), the conditional bias $\mathrm{b}\left(l_{m} \mid l_{0}\right)=\left\langle l_{m}-l_{0}\right\rangle$ and variance $\mathrm{V}\left(l_{m} \mid l_{0}\right)=\left\langle\left(l_{m}-l_{0}\right)^{2}\right\rangle$ of MLE $l_{m}$, with the allowance for the anomalous errors, can be written down as follows:

$$
\begin{aligned}
& \mathrm{b}\left(l_{m} \mid l_{0}\right)=\mathrm{P}_{0} \mathrm{~b}_{0}\left(l_{m} \mid l_{0}\right)+\left(1-\mathrm{P}_{0}\right)\left[\left(\tilde{\Lambda}_{1}+\tilde{\Lambda}_{2}\right) / 2-l_{0}\right] \\
& \mathrm{V}\left(l_{m} \mid l_{0}\right)=\mathrm{P}_{0} \mathrm{~V}_{0}\left(l_{m} \mid l_{0}\right)+\left(1-\mathrm{P}_{0}\right) \\
& {\left[\left(\tilde{\Lambda}_{1}^{2}+\tilde{\Lambda}_{1} \tilde{\Lambda}_{2}+\tilde{\Lambda}_{2}^{2}\right) / 3-l_{0}\left(\tilde{\Lambda}_{1}+\tilde{\Lambda}_{2}\right)+l_{0}^{2}\right]}
\end{aligned}
$$

Here, $\quad \mathrm{b}_{0}\left(l_{m} \mid l_{0}\right), \quad \mathrm{V}_{0}\left(l_{m} \mid l_{0}\right), \quad P_{0}=P\left[\left|l_{m}-l_{0}\right| \leq 1\right]$ are, correspondingly, conditional bias, conditional variance and probability of a reliable estimate $l_{m}$ (26).

While determining $b_{0}\left(l_{m} \mid l_{0}\right), V_{0}\left(l_{m} \mid l_{0}\right)$ and $\mathrm{P}_{0}$, we will be limited to a condition of a high posterior accuracy when the output signal-to-noise ratio (SNR) $\mathrm{z}^{2}$ of the algorithm (26) is sufficiently great, i.e.

$\mathrm{z}^{2}=\left[\mathrm{S}\left(l_{0}\right)-\mathrm{S}_{N}\right]^{2} /\left\langle\mathrm{N}^{2}\left(l_{0}\right)\right\rangle=$

$\mu \mathrm{q}_{0}^{2}\left[\int_{-1 / 2}^{1 / 2} \frac{\mathrm{f}^{4}(\tilde{\gamma} \tilde{\mathrm{t}}) \mathrm{d} \tilde{\mathrm{t}}}{1+\mathrm{q}_{0} \mathrm{f}^{2}(\tilde{\gamma} \tilde{\mathrm{t}})}\right]^{2} / \int_{-1 / 2}^{1 / 2} \mathrm{f}^{4}(\tilde{\gamma} \tilde{\mathrm{t}}) \mathrm{d} \tilde{\mathrm{t}}>>1$.

The inequality (29) is valid with ratios (2) executed and when the values of $\mathrm{q}_{0}$ are not too small.

We will presuppose that $f(\tilde{\gamma} \tilde{\mathrm{t}})$ does not vanish in points $\tilde{\mathrm{t}}= \pm 1 / 2$, i.e. the useful signal (1) is discontinuous (for a continuous signal the results received in [5] can be used at their obvious generalization). Similarly to [6], it can be shown that MLE $l_{m}$ converges in a mean square to a true value of the estimated parameter $l_{0}$ with the increase of $\mathrm{z}^{2}$. Hereupon, for the definition of the characteristics of the reliable estimate $l_{m}$ under $z^{2}>>1$, it is sufficient to investigate the behavior of the functional $\mathrm{M}(l)(22)$ in a small neighborhood of the point $l=l_{0}$. We will designate $\Delta=\max \left\{\left|l_{1}-l_{0}\right|,\left|l_{2}-l_{0}\right|,\left|l_{1}-l_{2}\right|\right\}$. Then, taking into account the ratios (2) under $\Delta \rightarrow 0$, the following asymptotic expansions are true for Eqs. (23):

$$
\begin{aligned}
& \mathrm{S}(l)=\mu \mathrm{d}_{0}\left[\mathrm{G}_{14}(1 / 2)+\mathrm{g}_{41}(1 / 2) \min \left(0, l-l_{0}\right)-\right. \\
& \left.-\mathrm{g}_{41}(-1 / 2) \max \left(0, l-l_{0}\right)\right]+\mathrm{S}_{N}+\mathrm{o}(\Delta),
\end{aligned}
$$

$$
\begin{aligned}
& \left\langle\mathrm{N}\left(\mathrm{l}_{1}\right) \mathrm{N}\left(\mathrm{l}_{2}\right)\right\rangle=\mu \mathrm{N}_{0}^{2}\left\{\mathrm{G}_{40}(1 / 2)+\mathrm{g}_{42}(1 / 2) \min \right. \\
& \left(l_{1}-l_{0}, l_{2}-l_{0}\right)-\mathrm{g}_{42}(-1 / 2) \max \left(l_{1}-l_{0}, l_{2}-l_{0}\right)+ \\
& +\left[\mathrm{g}_{40}(1 / 2)-\mathrm{g}_{42}(1 / 2)\right]-\min \left(0, l_{1}-l_{0}, l_{2}-l_{0}\right)- \\
& \left.-\left[\mathrm{g}_{40}(-1 / 2)-\mathrm{g}_{42}(-1 / 2)\right] \max \left(0, l_{1}-l_{0}, l_{2}-l_{0}\right)\right\}+\mathrm{o}(\Delta),
\end{aligned}
$$

where $\mathrm{o}(\Delta)$ denotes the higher-order infinitesimal terms 
compared with $\Delta$ and $\mathrm{g}_{m n}(\tilde{\mathrm{t}}), \mathrm{G}_{m n}(\tilde{\mathrm{t}})$ are defined according to Eqs. (24).

Using results [9 and 10], on the basis of regular and noise functions properties (30) of solving statistics (22) for conditional bias $\mathrm{b}_{0}\left(l_{m} \mid l_{0}\right)$, conditional variance $\mathrm{V}_{0}\left(l_{m} \mid l_{0}\right)$ and probability of a reliable estimate $l_{m}$ we find $\mathrm{b}_{0}\left(l_{m} \mid l_{0}\right) \approx 0$,

$$
\begin{aligned}
& \mathrm{V}_{0}\left(l_{m} \mid l_{0}\right)=13\left\{\mathrm{f}^{4}(\tilde{\gamma} / 2) \mathrm{f}(-\tilde{\gamma} / 2)\left[1+\mathrm{q}_{0} \mathrm{f}^{2}(\tilde{\gamma} / 2)\right]^{2}\right\} \times \\
& \times\left\{\mathrm{f}^{4}(-\tilde{\gamma} / 2)+\mathrm{f}^{4}(\tilde{\gamma} / 2)\left[1+\mathrm{q}_{0} \mathrm{f}^{2}(-\tilde{\gamma} / 2)\right]^{2}\right\} / 8 \mu^{2} \mathrm{q}_{0}^{4} \\
& \mathrm{f}^{8}(\tilde{\gamma} / 2) \mathrm{f}^{8}(-\tilde{\gamma} / 2),
\end{aligned}
$$

$$
\begin{aligned}
& \mathrm{P}_{0}=\frac{\mathrm{z}\left(\psi_{1}+\psi_{2}\right)}{\mathrm{r}} \int_{1}^{\infty} \exp \left[-\frac{\hat{\mathrm{m}} \kappa}{\sqrt{2 \pi}} \exp \left(-\frac{\kappa^{2}}{2}\right)\right] \\
& \left\{\frac{\psi_{1}}{\psi_{1}+\psi_{2}} \exp \left[\frac{\psi_{1}^{2} z^{2}}{2}+\psi_{1} \mathrm{z}\left(\mathrm{z}-\frac{\kappa}{\mathrm{r}}\right)\right]\right\} \times \\
& \times \Phi\left[\frac{\kappa}{\mathrm{r}}-\mathrm{z}\left(\psi_{1}+1\right)\right]+\frac{\psi_{2}}{\psi_{1}+\psi_{2}} \exp \left[\frac{\psi_{2}^{2} z^{2}}{2}+\right. \\
& \left.+\psi_{2} \mathrm{z}\left(\mathrm{z}-\frac{\kappa}{\mathrm{r}}\right)\right] \Phi\left[\frac{\kappa}{\mathrm{r}}-\mathrm{z}\left(\psi_{2}+1\right)\right]- \\
& -\exp \left[\frac{\mathrm{z}^{2}\left(\psi_{1}+\psi_{2}\right)^{2}}{2}+\mathrm{z}\left(\psi_{1}+\psi_{2}\right)\left(\mathrm{z}-\frac{\kappa}{\mathrm{r}}\right)\right] \Phi \times \\
& \left.\times\left[\frac{\kappa}{\mathrm{r}}-\mathrm{z}\left(\psi_{1}+\psi_{2}+1\right)\right]\right\} \mathrm{d} \kappa,
\end{aligned}
$$

where $\hat{\mathrm{m}}=\mathrm{m}\left[\mathrm{g}_{42}(1 / 2)+\mathrm{g}_{42}(-1 / 2)\right] / 2 \mathrm{G}_{42}(1 / 2)$ is reduced length [6] of the prior limit of signal (1) appearance time, $\psi_{1}=2 \mathrm{~g}_{41}(1 / 2) \mathrm{G}_{40}(1 / 2) / \mathrm{G}_{41}(1 / 2)\left[\mathrm{g}_{40}(1 / 2)+\mathrm{g}_{42}(-1 / 2)\right]$, $\psi_{2}=2 \mathrm{~g}_{41}(-1 / 2) \mathrm{G}_{40}(1 / 2) / \mathrm{G}_{41}(1 / 2)\left[\mathrm{g}_{40}(-1 / 2)+\mathrm{g}_{42}(-1 / 2)\right]$, $\mathrm{r}=\sqrt{\mathrm{G}_{40}(1 / 2) / \mathrm{G}_{42}(1 / 2)}$. Accuracy of formulas (31) increases with $\mu$ (23), z (29) and $\mathrm{m}$ (27).

If $\mathrm{f}(\mathrm{t}) \equiv 1$, then from Eqs. (31) we receive known expressions for conditional bias, conditional variance and probability of a reliable estimate for appearance time MLE $\lambda_{m}$ of a HF random pulse (1), (14) with rectangular envelope [3].

\section{Detection of the random Gaussian pulse with unknown appearance time}

Now let us consider a detection problem of the HF random pulse (1), (14) with unknown appearance time $\lambda_{0}$. As it is well known from [6], MLR makes a decision about the presence of a useful signal in terms of the comparison of the absolute (greatest) maximum value of the logarithm of FLR (21), with a threshold $c$ which is chosen according to the set optimality criterion of detection. Therefore, the magnitude

$$
\mathrm{L}=\sup _{\lambda \in\left[\Lambda_{1}, \Lambda_{2}\right]} \mathrm{L}(\lambda)=\sup _{\lambda \in\left[\Lambda_{1}, \Lambda_{2}\right]} \mathrm{M}(\lambda)
$$

should be compared with a threshold $c$. Here $\mathrm{M}(\lambda)$ is determined from Eq. (22).
The block diagram of the detection algorithm (32) of the random pulse can be obviously received through the block diagram presented in Fig. 1 by substituting extremator 5 for solving device, which analyzes input realization $\mathrm{M}(\lambda)$ (22) within the interval $\lambda \in\left[\Lambda_{1}, \Lambda_{2}\right]$ and makes a decision about presence or absence of a signal (1), (14), comparing an absolute maximum of realization $\mathrm{M}(\lambda)$ with a threshold $c$.

Let us find characteristics of random pulse detector (32). As such characteristics we will use type I (false-alarm) and II (signal missing) error probabilities [1, 2, 4, 6, etc.]. The starting point is that the useful signal is absent. Then the false-alarm probability $\alpha$ can be determined as follows

$$
\begin{aligned}
& \alpha=\mathrm{P}\left[\sup _{\lambda \in\left[\Lambda_{1}, \Lambda_{2}\right]} \mathrm{M}(\lambda)>\mathrm{c} \mid \mathrm{x}(\mathrm{t})=\mathrm{n}(\mathrm{t})\right]= \\
& =\mathrm{P}\left[\sup _{l \in\left[\tilde{\Lambda}_{\left.1, \tilde{L}_{2}\right]}\right.} \stackrel{\tilde{\mathrm{M}}}{ }(l)>\mathrm{u} \mid \mathrm{x}(\mathrm{t})=\mathrm{n}(\mathrm{t})\right] .
\end{aligned}
$$

Here, $\stackrel{2}{\mathrm{M}}(l)\left[\mathrm{M}(l)-\mathrm{S}_{N}\right] / \sigma_{N}, \sigma_{N}=\mathrm{N}_{0} \sqrt{\mu \mathrm{G}_{42}(1 / 2)}$, and $\mathrm{u}=\left(\mathrm{c}-\mathrm{S}_{N}\right) / \sigma_{N}$ is normalized threshold.

As follows from [4], (25), the functional $\stackrel{\tilde{M}}{(}(l)$ is asymptotically Gaussian (under $\mu \rightarrow \infty$ ) with zero mathematical expectation and unit dispersion. According to [11 and 12], while increasing the threshold $c$, the distribution of number of outliers over level $c$ by stationary centered Gaussian random process converges to the Poisson's law with threshold $c$ growth. On the basis of results [4 and 9] it allows to write down asymptotically (under $\mathrm{m} \rightarrow \infty, \mathrm{c} \rightarrow \infty$ ) exact expression for falsealarm probability (33) as

$\alpha= \begin{cases}1-\exp \left[-\frac{\hat{m} u}{\sqrt{2 \pi}} \exp \left(-u^{2} / 2\right)\right] & , u \geq 1, \\ 1, & u<1 .\end{cases}$

Let suppose now that the signal (1), (14) is present on the detector input. Then the missing probability $\beta$ will be defined as $[1,2,4,6$, etc]

$$
\begin{aligned}
& \beta=P\left[\sup _{\lambda \in\left[\Lambda_{1}, \Lambda_{2}\right]} \mathrm{M}(\lambda)<\mathrm{c} \mid \mathrm{x}(\mathrm{t})=\mathrm{s}(\mathrm{t})+\mathrm{n}(\mathrm{t})\right]= \\
& =\mathrm{P}\left[\sup _{l \in\left[\Lambda_{1}, \Lambda_{2}\right]} \mathrm{M}(l)<\mathrm{c} \mid \mathrm{x}(\mathrm{t})=\mathrm{s}(\mathrm{t})+\mathrm{n}(\mathrm{t})\right] .
\end{aligned}
$$

Using Eqs. (23)-(25) it is easy to show that if condition (27) is satisfied then values of the absolute maxima of functional $\mathrm{M}(l)$ are approximately statistically independent in subareas $\Gamma_{S}$ and $\Gamma_{N}$. The specified property allows similarly [4 and 9] to present Eq. (35) in a kind of

$\beta=F_{N}(u) F_{S}(u / r)$,

Here, $\mathrm{F}_{S}(\mathrm{u})=\mathrm{P}\left\{\sup _{l \in \Gamma_{s}} \stackrel{\circ}{\mathrm{M}}(l) / \sigma_{s}<\mathrm{u}\right\}$, 
$\mathrm{F}_{N}(\mathrm{u})=\mathrm{P}\left\{\sup _{l \in \Gamma_{N}} \stackrel{\circ}{\mathrm{M}}(l) / \sigma_{N}<\mathrm{u}\right\}$ are distribution functions of the normalized absolute maxima of centered functional $\stackrel{\circ}{\mathrm{M}}(l)=\mathrm{M}(l)-\mathrm{S}_{N}$ within intervals $\Gamma_{S}$ and $\Gamma_{N}$ correspondingly, $\sigma_{s}=N_{0} \sqrt{\mu G_{40}(1 / 2)}, \mathrm{r}=\sigma_{S} / \sigma_{N}=\sqrt{\mathrm{G}_{40}(1 / 2) / \mathrm{G}_{42}(1 / 2)}$.

When condition (27) holds

$$
\begin{aligned}
& \mathrm{P}\left\{\sup _{l \in \Gamma_{N}} \stackrel{\circ}{\mathrm{M}}(l) / \sigma_{N}<\mathrm{u}\right\} \approx \mathrm{P}\left\{\sup _{l \in \Gamma} \stackrel{\circ}{\mathrm{M}}(l) / \sigma_{N}<\mathrm{u} \mid\right. \\
& \mid \mathrm{x}(\mathrm{t})=\mathrm{n}(\mathrm{t})\},
\end{aligned}
$$

and approximation

$\mathrm{F}_{\mathrm{N}}(\mathrm{u})=1-\alpha= \begin{cases}\exp \left[-\frac{\hat{\mathrm{m} u}}{\sqrt{2 \pi}} \exp \left(-\frac{\mathrm{u}^{2}}{2}\right)\right], \mathrm{u} \geq 1, \\ 0, & \mathrm{u}<1 .\end{cases}$

can be used for function $\mathrm{F}_{\mathrm{N}}(\mathrm{u})$.

For probability $\mathrm{F}_{\mathrm{S}}(\mathrm{u})$, using results [4 and 9 ] and taking into account Eqs. (23), we find

$$
\begin{aligned}
& F_{S}(u)=\Phi(u-z)-\exp \left[\psi_{1}^{2} z^{2} / 2+\psi_{1} z(z-u)\right] \times \\
& \times \Phi\left[u-z\left(\psi_{1}+1\right)\right]-\exp \left[\psi_{2}^{2} z^{2} / 2+\psi_{2} z(z-u)\right] \times \\
& \times \Phi\left[u-z\left(\psi_{2}+1\right)\right]+\exp \left[z^{2}\left(\psi_{1}+\psi_{2}\right)^{2} / 2+\right. \\
& \left.+z\left(\psi_{1}+\psi_{2}\right)(z-u)\right] \Phi\left[\kappa-z\left(\psi_{1}+\psi_{2}+1\right)\right] .
\end{aligned}
$$

Now, applying the approximations (37), (38) to the Eq. (36) we have finally

$$
\begin{aligned}
& \beta=\exp \left[-\frac{\hat{m} u}{\sqrt{2 \pi}} \exp \left(-\frac{\mathrm{u}^{2}}{2}\right)\right]\left\{\Phi\left(\frac{\mathrm{u}}{\mathrm{r}}-\mathrm{z}\right)-\right. \\
& -\exp \left[\frac{\psi_{1}^{2} z^{2}}{2}+\psi_{1} \mathrm{z}\left(\mathrm{z}-\frac{\mathrm{u}}{\mathrm{r}}\right)\right] \times \Phi\left[\frac{\mathrm{u}}{\mathrm{r}}-\mathrm{z}\left(\psi_{1}+1\right)\right]- \\
& -\exp \left[\frac{\psi_{2}^{2} z^{2}}{2}+\psi 2 z\left(z-\frac{\mathrm{u}}{\mathrm{r}}\right)\right] \Phi\left[\frac{\mathrm{u}}{\mathrm{r}}-\mathrm{z}(\psi 2+1)\right]+ \\
& +\exp \left[\frac{\mathrm{z}^{2}\left(\psi_{1}+\psi_{2}\right)^{2}}{2}+\mathrm{z}\left(\psi_{1}+\psi_{2}\right)\left(\mathrm{z}-\frac{\mathrm{u}}{\mathrm{r}}\right)\right] \times \\
& \left.\times \Phi\left[\frac{\mathrm{u}}{\mathrm{r}}-\mathrm{z}\left(\psi_{1}+\psi_{2}+1\right)\right]\right\},
\end{aligned}
$$

if $u \geq 1$, and $\beta \approx 0$ if $u<1$. The accuracy of the formula (39) increases with $\mathrm{u}, \mathrm{m}, \mu, \mathrm{z}$.

Supposing in Eqs. (34), (39) $\mathrm{f}(\mathrm{t}) \equiv 1$, we receive expressions for false-alarm and missing probabilities at the reception of a HF random pulse (1), (14) with rectangular envelope and unknown appearance time [4 and 9].

\section{Results of statistical modeling}

For the purpose of an establishment of the applicability borders of the asymptotically exact formulas for the detection and estimation characteristics, we applied the MLR statistical computer modeling.

In the process of modeling, we followed the technique, introduced in [9], and formed the samples of the normalized functional $\tilde{\mathrm{M}}(l)=\mathrm{M}(\lambda) / \mathrm{N}_{0}(22)$ within an interval $\left[\tilde{\Lambda}_{1}, \tilde{\Lambda}_{2}\right]$ with a step $\Delta l=0.01$, both in the presence and in the absence of the signal (1), (14). And thus the relative mean square error of step approximations of continuous realizations of process $\tilde{\mathrm{M}}(l)$ in terms of the generated discrete samples did not exceed $10 \%$. For each realization of $\tilde{\mathrm{M}}(l)$ we compared the value of the greatest maximum sample of the functional (22) with the normalized threshold $\mathrm{c} / \mathrm{N}_{0}$ and found the false-alarm and missing probabilities. Besides, according to (26), we determined the estimate $l_{m}$ and its variance.

Some results of statistical modeling for $l_{0}=\left(\tilde{\Lambda}_{2}+\tilde{\Lambda}_{1}\right) / 2$, $\tilde{\Lambda}_{1}=1 / 2, \quad \tilde{\Lambda} 2=\mathrm{m}+1 / 2 \quad$ and $\quad \mathrm{f}(\tilde{\mathrm{t}})=\exp \left(-\tilde{\gamma}^{2} \tilde{\mathrm{t}}^{2}\right)$, $\tilde{\gamma}=1$ are shown in Figs. 2a $-4 a$, while for $\mathrm{f}(\tilde{\mathrm{t}})=1-\tilde{\gamma}|\tilde{\mathrm{t}}|$, $\tilde{\gamma}=1 / 4$ - in Figs. $2 \mathrm{~b}-4 \mathrm{~b}$. Each experimental value in Figs. 2 - 4 was obtained as a result of the processing of no less than $10^{4}$ realizations of the solving statistics $\tilde{M}(1)$. In this case, with probability of 0.9 confidence intervals boundaries deviate from experimental values no more than for $5 \ldots 10 \%$.

In Figs. 2 the theoretical dependences of the normalized conditional variance $\mathrm{V}_{1}\left(\mathrm{q}_{0}\right)=12 \mathrm{~V}\left(l_{m} \mid l_{0}\right) / \mathrm{m}^{2}$ (28) of MLE $l_{\mathrm{m}}$, calculated in view of $m=20$ and with the allowance for anomalous errors, are drawn as solid lines. The dashed lines in Figs. 2 show the analogous dependences of the normalized conditional variance $\mathrm{V}_{1}\left(\mathrm{q}_{0}\right)=12 \mathrm{~V}\left(l_{m} \mid l_{0}\right) / \mathrm{m}^{2}$ (31) of reliable MLE $l_{\mathrm{m}}$. Curves 1 correspond to $\mu=50$, curves 2 to $\mu=100$, curves 3 to $\mu=200$. In Figs. 2 the experimental values for $\mu=50,100$ and 200 are denoted by rectangles, crosses, and rhombuses (for variance $\mathrm{V}_{l}$ of MLE $l_{\mathrm{m}}$ with the allowance for anomalous errors), respectively, as well as by plus signs, circles and triangles (for variance $\mathrm{V}_{0 l}$ of reliable $\mathrm{MLE} l_{\mathrm{m}}$ ).

In Figs. 3 the theoretical dependences (34) of false-alarm probability $\alpha(\mathrm{u})$ (33) are plotted. The curve 1 corresponds to $\mathrm{q}=0.1$ and curve 2 to $\mathrm{q}=4$. Parameter quantity $\mathrm{m}$ assumed equal 20. Experimental values of false-alarm probability (33) for $m=20$ are shown in Figs. 3 by rectangles and crosses (for $\mathrm{q}=0.1$ and $\mu=50$ or $\mu=200$ ), respectively, as well as rhombuses and circles (for $\mathrm{q}=4$ and $\mu=50$ or $\mu=200$ ).

At last, in Figs. 4 for $m=20$ the theoretical dependences of missing probability $\beta\left(q_{0}\right)$ (35) are represented. The curve 1 is calculated with the formula (39) for $\mu=50$, curve 2 for $\mu=100$ and curve 3 for $\mu=200$. The threshold quantity u was determined from Eq. (34) by Neumann-Pirson criterion, according to the preselected level of false-alarm probability equal to 0.01 . Experimental values of the missing probability (35) for $\mu=50,100$ and 200 are designated in Figs. 4 by rectangles, crosses, and rhombuses respectively.

In terms of the received results, we can draw the following conclusions.

As is evident from Figs. 2 - 4, the theoretical dependences both of the detection and of the appearance time estimation of the random pulse (1), (14), with the allowance for the anomalous errors, approximate the experimental results in a satisfactory manner, at least, for $m \geq 20, \mu \geq 50, z \geq 1.5 \ldots 2$, 

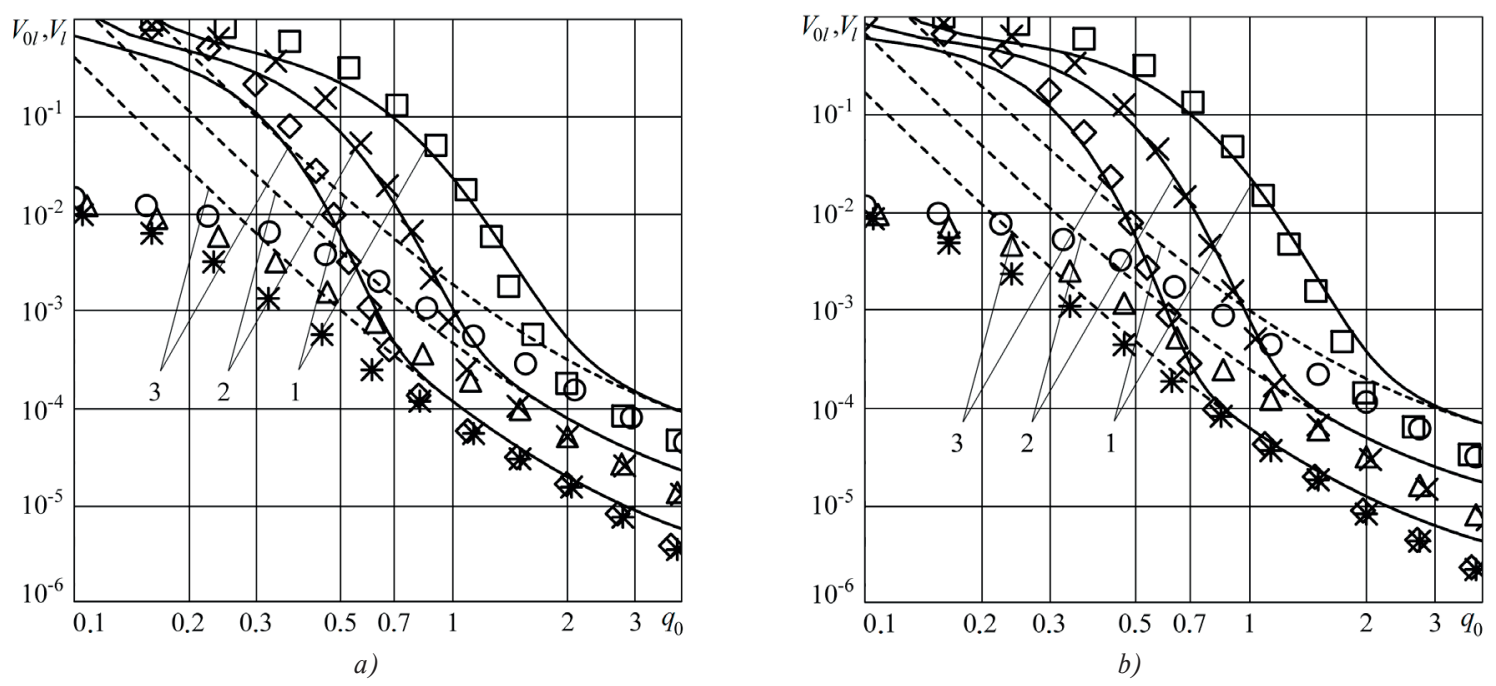

Fig. 2 The theoretical and experimental dependences of normalized variance of appearance time estimate
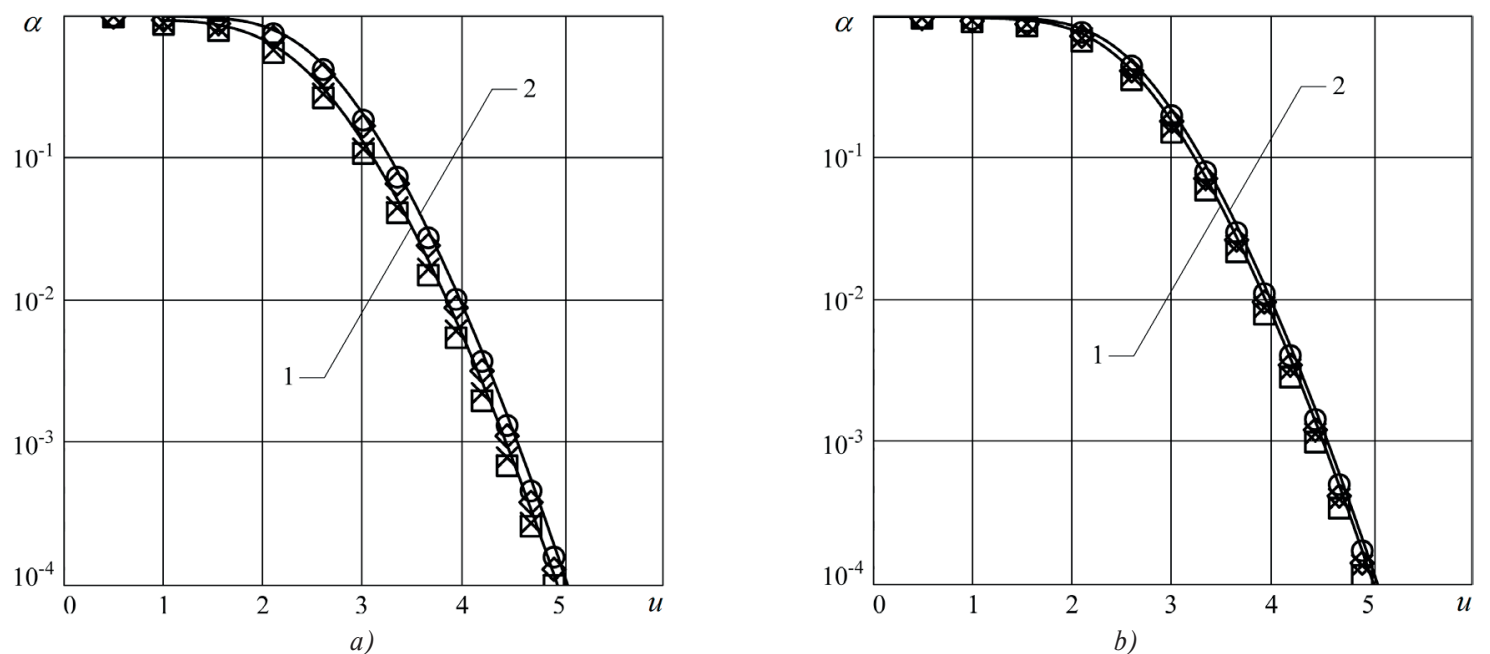

Fig. 3 The theoretical and experimental dependences of false-alarm probability
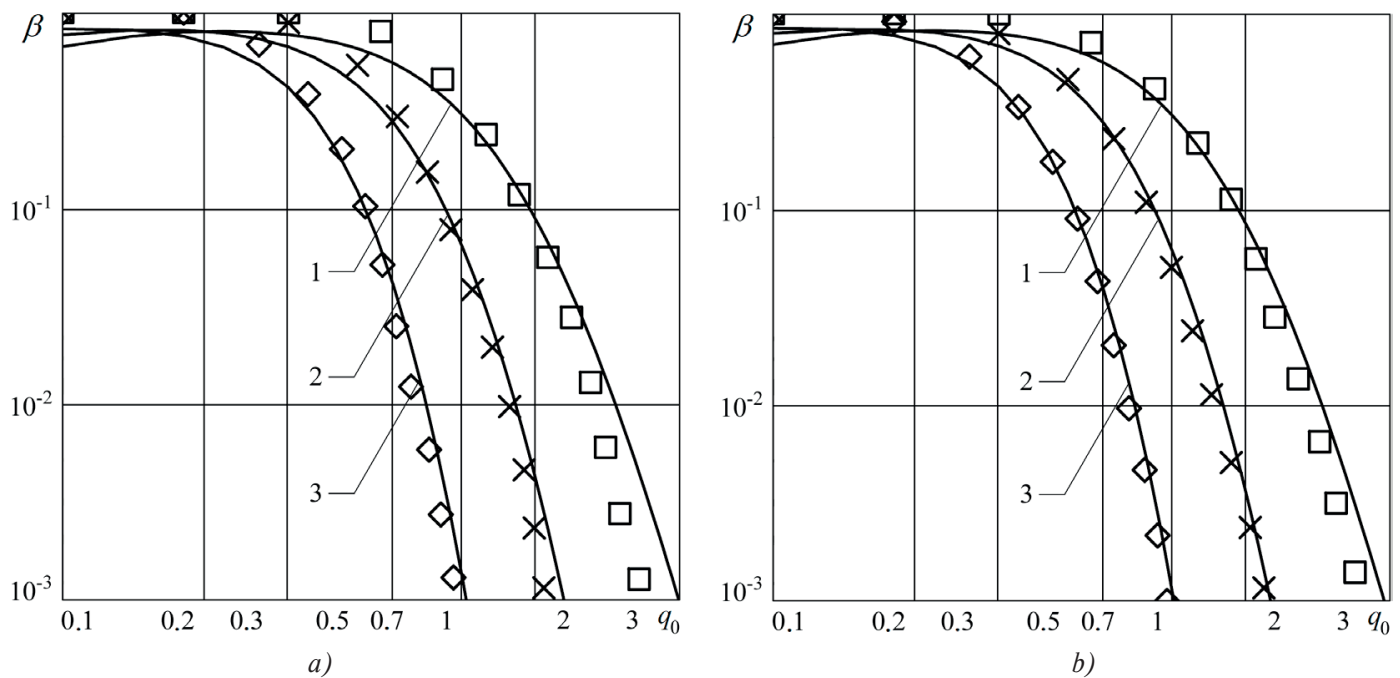

Fig. 4 The theoretical and experimental dependences of missing probability 
$\left(\mathrm{f}_{\max }-\mathrm{f}_{\min }\right) / \mu<4 \cdot 10^{-3}$. Here, $\mathrm{f}_{\min }=\min \mathrm{f}(\mathrm{t})$ and $\mathrm{f}_{\max }=1$ are minimum and maximum values of the function $f(t)$.

As $\mathrm{q}_{0}$ decreases when the SNR $\mathrm{z} \leq 5 \ldots .6$, the probability $\mathrm{P}_{\mathrm{a}}=1-\mathrm{P}_{0}$ of the anomalous errors increases significantly and approaches 1 . This leads to a jump-like (compared with the case of reliable estimate) increase in the variance of the appearance time estimate (so-called threshold effects occur). As $\mathrm{q}_{0}$ increases, when $\mathrm{z}>5 \ldots 6$, the variance $\mathrm{V}_{l}$ converges to the variance $\mathrm{V}_{0}$, and the estimate $l_{m}(26)$ becomes reliable with the probability close to 1. From Eqs. (28), (31) and Figs. 2 follows that the minimum (threshold) value of the parameter $\mathrm{q}_{0}$, at which the influence of the anomalous errors on the accuracy of MLE of the signal (1), (14) appearance time can be still neglected, decreases with $\mu$ and increases with $\mathrm{m}$.

For $z<2 \ldots 3$ the theoretical dependences of variance $V_{0 /}$ of the reliable MLE (26) deviate from experimental ones substantially, as are found without finite length of the interval $\Gamma_{S}$ of the reliable MLE like values. Departing of theoretical dependences $\mathrm{V}_{0}\left(l_{\mathrm{m}} \mid l_{0}\right)$ (31), $\mathrm{V}\left(l_{\mathrm{m}} \mid l_{0}\right)$ (28) from experimental data is observed under high SNR when $\mathrm{q}>2 \ldots 3$ too. This is due to the fact that Eq. (31) for the variance $\mathrm{V}_{0}\left(l_{\mathrm{m}} \mid l_{0}\right)$ of the reliable estimate of the appearance time is obtained ignoring the errors in estimating the order of correlation time of the random substructure of the pulsed signal (1), (14). Consequently, the error of Eqs. (28), (31) becomes significant, as the relative variance of the MLE decreases to a magnitude of order $\mu^{-2}$.

\section{Conclusion}

For the synthesis of the algorithms of the statistical analysis of random pulsed signals with the arbitrary-function envelope, the approach neglecting the values of an order of correlation time of a pulse random substructure is effective. The given approach makes it possible to receive detectors and measurers of the random pulses with the unknown parameters sufficiently simply realized in practice.

Quality of the synthesized processing algorithms of the pulsed signals with the unknown discontinuous parameters can be theoretically estimated by means of the local Markov approximation method based on the representation of the solving statistics increments by the Markov random process of diffusion type in a small neighborhood of the point of the true value of the unknown parameter [9]. As a result, it is possible to write down the closed analytical expressions for detection and estimation characteristics having acceptable accuracy for SNR being more than $1 \ldots 2$, and that was proved by the results of the statistical modeling.

The results of the study are of a general importance. They can be used in radio physical measurements while processing the random pulses of optical, acoustic, electromagnetic and other origins, in the systems information transmission and processing and in the systems of the technological processes monitoring and control, and also in other fields of science and technology, dealing with registration and measurement of random processes.

\section{Acknowledgement}

The reported study was supported by the Russian Science Foundation (research project No. 14-49-00079).

\section{References}

[1] VAN TREES, H. L.: Detection, Estimation, and Modulation Theory, Part III, Wiley: New York, 1971.

[2] Problems of the Statistical Radiolocation Theory [in Russian], Edited by G. P. Tartakovsky, Sovetskoe Radio, Moscow, 1963.

[3] TRIFONOV, A. P., ZAKHAROV, A. V.: Reception of Signals with Unknown Delay in the Presence of Modulating Noise, Radioelectronics and Communications Systems, vol. 29, No. 4, 1986, pp. 32-36.

[4] TRIFONOV, A. P., NECHAEV, E. P., PARFENOV, V. I.: Detection of Stochastic Signals with Unknown Parameters [in Russian], Voronezh State University, Voronezh, 1991.

[5] TRIFONOV, A. P., PARFENOV, V. I.: Theoretical and Experimental Investigations of a Maximum-Likelihood Receiver of a Random Pulse with an Unknown Arrival Time, J. of Communications Technology and Electronics, vol. 43, No 7, 1998, pp. 769-775.

[6] TRIFONOV, A. P., SHINAKOV, Yu. S.: Joint Discrimination of Signals and Estimation of their Parameters against Background [in Russian], Radio i Svyaz', Moscow, 1986.

[7] CHERNOYAROV, O. V., BREZNAN, M., TEREKHOV A. V.: Restoration of Deterministic and Interference Distorted Signals and Images with Use of the Generalized Spectra in Bases of Orthogonal Polynomials and Functions, Communications - Scientific Letters of the University of Zilina, vol. 15, No. 2A, 2013, pp. 71-77.

[8] TARTAKOVSKY, G. P.: Synthesis of a Heterodyne Light Signal Receiver [in Russian], Problemy Peredachi Informatsii, vol. 1, No. 3, 1965, pp. 56-70. 
[9] CHERnOYAROV, O. V., SAI Si THU MIN, SAlniKOVA, A.V., SHAKHTARIN, B. I., ARTEMENKO, A. A.: Application of the Local Markov Approximation Method for the Analysis of Information Processes Processing Algorithms with Unknown Discontinuous Parameters, Applied Mathematical Sciences, vol. 8, No 90, 2014, pp. 4469-4496.

[10] Chernoyarov, O. V., SAlnikova, A. V., ROZANOV, A. E., MARCOKOVA, M.: Statistical Characteristics of the Magnitude and Location of the Greatest Maximum of Markov Random Process with Piecewise Constant Drift and Diffusion Coefficients, Applied Mathematical Sciences, vol. 8, No 147, 2014, pp. 7341-7357.

[11] PICKANDS, J.: Upcrossing Probabilities for Stationary Gaussian Process, Trans. Amer. Math. Soc., vol. 145, No. 11, 1969, p. 51-73.

[12] QUALlS, C., WATANABE, H.: Asymptotic Properties of Gaussian Processes, Ann. on Math. Statist., vol. 3, No. 2, 1972, p. 580596. 